\title{
A general optimization-based approach for thermal processes modeling
}

\author{
Paweł Drąg \\ Department of Control Systems and Mechatronics \\ Wrocław University of Science and Technology \\ Wybrzeże Wyspiańskiego 27, 50-370 Wrocław, Poland \\ Email: pawel.drag@pwr.edu.pl
}

\author{
Krystyn Styczeń \\ Department of Control Systems and Mechatronics, \\ Wrocław University of Science and Technology \\ Wybrzeże Wyspiańskiego 27, 50-370 Wrocław, Poland \\ Email: krystyn.styczen@pwr.edu.pl
}

\begin{abstract}
In the article a new optimization approach for thermal processes modeling has been presented. In the designed method, the considered process is monitored by a measurement system with a thermal camera. Then, a spatio-temporal dynamics is discretized and transformed into a large-scale optimization problem with differential-algebraic constraints. To preserve the process dynamics in the assumed range, variability constraints have been imposed. Finally, a new interior-point optimization algorithm has been designed to solve the optimization problem with the variability constraints. The applicability of the new approach has been investigated experimentally.
\end{abstract}

Index Terms - variability constraints, DAE systems, nonlinear optimization, thermal processes, thermal camera,

\section{INTRODUCTION}

O PTIMIZATION and control of thermal processes is a complex issue, which has a large impact on various branches of industry. Therefore, a general solution procedure is consisted on the following steps:

1) Thermal camera-based measurement system to perform observations of the considered surface [15].

2) The obtained measurements represent the spatiotemporal dynamics of the process. Therefore, it is needed to design an appropriate model of the considered process [17].

3) A direct transcription method enables us to apply a large-scale nonlinear optimization algorithms to solve a model optimization problem.

The complexity of the considered issue motivates a recently progress in the optimization of many real-life technological processes. In the work [18] a three-dimensional numerical model using enthalpy technique to describe the solidification of phase change material has been developed. Therefore, the effect of geometrical parameters on the thermal performance of latent heat thermal energy storage system has been studied. Finally, the optimum system geometry could be identified. Mei and Xia [14] designed a multi-input-multi-output (MIMO) model predictive control (MPC) for a direct expansion air conditioning system to improve an indoor thermal comfort, as well as air quality. Moreover, the energy consumption has been minimized. The considered direct expansion air conditioning system has been described by nonlinear algebraic equations. The designed procedure has been verified by obtained simulation results. In the article [11] a heat management in optimization of highly exothermic reactions during gasphase olefin polymerization in fluidized bed reactors has been discussed. Moreover, a high speed infrared (IR) camera and a visual camera have been coupled to present the hydrodynamic and thermal behavior of a pseudo-2D fluidized bed. The applied infrared/visual camera technique generated detailed information on the thermal behavior of the bed and enabled to optimize a combined computational fluid dynamics and discrete element model. Mariani et al. [12] considered a gassolid cyclone separator used in a complex cement production plant. The objective of the study was aimed at optimization of the performance evaluation, as well as the cyclone separator in terms of particle separation and heat transfer efficiencies. The losses of the pressure were treated as the additional technological constraints. Bhaduri et al. [2] reported results from process optimization experiments aimed at investigating the influence of laser fluence and pulse overlap parameters on resulting workpiece surface roughness following laser polishing of planar 3D printed stainless steel (SS316L) specimens. The optimized laser polishing technology was implemented for serial finishing of structured 3D printed mesoscale SS316L components. Finally, Uribe-Soto et al. [19] presented recent approaches to significantly reduce or avoid $\mathrm{CO}_{2}$ emissions by a designed process optimization procedure.

A detailed analysis of the thermal processes is possible by efficient numerical optimization algorithms. Among the commonly known nonlinear optimization algorithms, the family of internal point methods is of a significant importance. Recently, an interior-point trust-funnel algorithm for solving large-scale nonlinear optimization problems has been presented in [5]. The designed method achieves global convergence guarantees by combining a trust-region methodology with a funnel mechanism. Moreover, it has a capability to solve problems with equality, as well as inequality constraints. An efficient primaldual interior-point algorithm using a new non-monotone line search filter method was presented in [20]. The designed nonmonotone line search technique has been introduced to lead to relaxed step acceptance conditions and improved convergence performance. Klintberg and Gros [10] designed an interior point method with an inexact factorization technique for optimal control of systems described by Differential-Algebraic Equations (DAEs). A class of convex optimization problems, 
where both the objective function and the constraints have a continuous dependence on time, have been considered in [9]. The designed method utilized a time-varying constraint slack and a prediction-correction structure that relies on time derivatives of functions and constraints and Newton steps in the spatial domain. Zorkaltsev [21] discussed a family of interior point algorithms for linear programming problems. In these algorithms, entering the feasible solution region of the original problem has been considered as an optimization process of a new extended problem. To obtain a solution of a large-scale optimization problem with a considerable time, Cao et al. [4] proposed an augmented Lagrangian interior-point approach for general NLP problems that solves in parallel on a Graphics Processing Unit (GPU).

The presented literature research indicates, that thermal process modeling with thermal camera-based approach, as well as with an application of a nonlinear optimization methods, is nowadays under intensive investigations. Therefore, the article is aimed at presentation of a general procedure, which enables us to optimize the temperature distribution model. The new issues introduced into optimization task are the technological constraints imposed on a temperature variability. The designed procedure is independent on a considered process and is consisted on measurement system, as well as an interior point optimization procedure.

This work is constructed as follows. In Section 2 the problem was introduced. The main parts of the measurement system, as well as data structures were presented. Moreover, the main solution idea was proposed. In Section 3 the temperature distribution control problem was transformed into a largescale nonlinear optimization problem. The variability of the state trajectories are treated as additional decision variables. Finally, the new interior-point optimization algorithm for solving dynamic optimization tasks with variability constraints was designed in Section 4. In Section 5 the presented considerations were practically illustrated. The article was concluded in Section 6.

\section{THE PROBLEM STATEMENT}

The task considered in this work is to find such parameters of the surface temperature distribution model $\widetilde{T}\left(x, y, \mathbf{v}_{\mathbf{p}}, t\right)$, that for a given time interval

$$
t \in\left[\begin{array}{ll}
t_{0} & t_{F}
\end{array}\right]
$$

the results obtained by the model simulations are appropriate to the given measurements

$$
\min _{\mathbf{v}_{\mathbf{p}}} \int_{t_{0}}^{t_{f}} \int_{x_{\min }}^{x_{\max }} \int_{y_{\min }}^{y_{\max }}\left(T^{\star}(x, y, t)-\widetilde{T}\left(x, y, \mathbf{v}_{\mathbf{p}}, t\right)\right)^{2} d y d x d t
$$

or

$$
\min _{\mathbf{v}_{\mathbf{p}}} \int_{t} \iint_{S}\left(T^{\star}(x, y, t)-\widetilde{T}\left(x, y, \mathbf{v}_{\mathbf{p}}, t\right)\right)^{2} d S d t
$$

where $S=\left[\begin{array}{ll}x_{\min } & x_{\min }\end{array}\right] \times\left[\begin{array}{ll}y_{\min } & y_{\min }\end{array}\right]$ denotes a range of the considered surface, an observation time range $t \in\left[\begin{array}{ll}t_{0} & t_{F}\end{array}\right]$, $T^{\star}(x, y, t)$ denotes a wanted temperature distribution on the surface at time interval $t \in\left[\begin{array}{ll}t_{0} & t_{F}\end{array}\right], \widetilde{T}\left(x, y, \mathbf{v}_{\mathbf{p}}, t\right)$ is the model of the temperature distribution for a given vector parameters $\mathbf{v}_{\mathbf{p}} \in \mathcal{R}^{n_{\mathbf{v}_{\mathbf{p}}}}$

As one can observe, the process is characterized by the spatio-temporal dynamics. Therefore, the value of measured temperature is dependent on the time, as well as on the values of geometrical coordinates. To reduce the number of independent variables, the spatial discretization approach was applied. This step enables us to divide the considered surface into an assumed number of cells. Therefore, each cell can be characterized by the values of geometrical coordinates.

Assumption 2.1: The considered surface $S$ can be partitioned into a given number of homogeneous cells

$$
c_{x, y}=\left[\begin{array}{ll}
x-\Delta x & x+\Delta x
\end{array}\right] \times\left[\begin{array}{ll}
y-\Delta y & y+\Delta y
\end{array}\right]
$$

Therefore, each cell $c_{x, y}$ can be described by a function dependent only on the time $t$

$$
c_{x, y} \equiv c_{x, y}(t) .
$$

This approach was presented on the Figure 1. The size of each cell is dependent on a discretization level. The reasonable size of the cell should be equal or bigger than size of a pixel. In the measurement system the thermal imaging camera FLIR A615 was used. The specification of the used hardware was presented in the Table I.

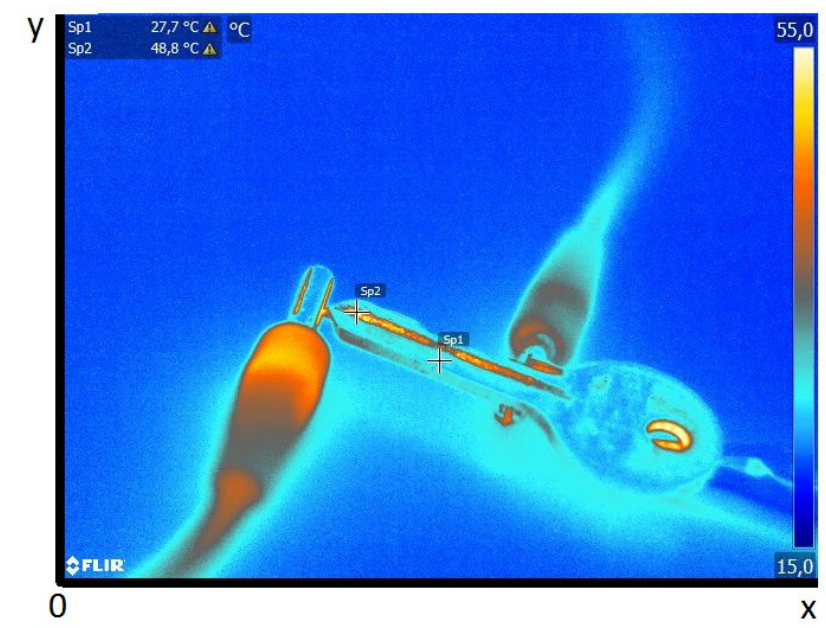

Fig. 1. An example of a thermal process.

Because each cell can be described by an exactly oneindependent-variable function, therefore a wide class of possible model functions can be indicated. One of the most general family of such functions are the index- 1 differential-algebraic models

$c_{x, y}(t)=\left\{\begin{aligned} \dot{z}_{x, y, d}(t) & =F_{x, y}\left(z_{x, y, d}(t), z_{x, y, a}(t), \mathbf{v}_{\mathbf{p}}, t\right) \\ 0 & =G_{x, y}\left(z_{x, y, d}(t), z_{x, y, a}(t), \mathbf{v}_{\mathbf{p}}, t\right)\end{aligned}\right.$ 
TABLE I

THE THERMAL IMAGING CAMERA FLIR A615 SPECIFICATION.

\begin{tabular}{ll}
\hline \hline Image frequency & $50 \mathrm{~Hz}$ \\
$\begin{array}{l}\text { Focal Plane Array (FPA)/ } \\
\text { Spectral range }\end{array}$ & $\begin{array}{l}\text { Uncooled microbolometer / } \\
7.5-14 \mu \mathrm{m}\end{array}$ \\
$\begin{array}{ll}\text { IR resolution } \\
\text { Detector time constant }\end{array}$ & $840 \times 480$ pixels \\
$\begin{array}{l}\text { Object temperature } \\
\text { range }\end{array}$ & $\begin{array}{l}-20 \text { to }+150^{\circ} \mathrm{C} \\
+100 \text { to } 650^{\circ} \mathrm{C} \\
+300 \text { to }+2000^{\circ} \mathrm{C}\end{array}$ \\
\hline
\end{tabular}

where $z_{x, y, d}(t) \in \mathcal{R}^{n_{z_{x, y, d}}}$ denotes a differential state variable of the cell $c_{x, y}, z_{x, y, a}(t) \in \mathcal{R}^{n_{z_{x, y}, a}}$ is an algebraic state variable of the cell $c_{x, y}, \mathbf{v}_{\mathbf{p}} \in \mathcal{R}^{n_{\mathbf{v}}}$ denotes a vector of the model parameters. Moreover, two vector-valued functions are considered

$$
\begin{aligned}
F_{x, y}: \mathcal{R}^{n_{z_{x}, y}} \times \mathcal{R}^{n_{z_{x, y}, a}} \times \mathcal{R}^{n_{\mathbf{v}_{\mathbf{p}}}} \times \mathcal{R} \rightarrow \mathcal{R}^{n_{z_{x, y}, d}} \\
G_{x, y}: \mathcal{R}^{n_{z_{x, y}, d}} \times \mathcal{R}^{n_{z_{x, y}, a}} \times \mathcal{R}^{n_{\mathbf{v}_{\mathbf{p}}}} \times \mathcal{R} \rightarrow \mathcal{R}^{n_{z_{x, y}, a}} .
\end{aligned}
$$

The presented approach enables us to describe each cell $c_{x, y}$ of the surface by a vector of descriptor variables $\mathbf{z}_{x, y}(t)$

$$
c_{x, y}(t)=\left\{\begin{aligned}
\dot{z}_{x, y, d}(t) & =F_{x, y}\left(\mathbf{z}_{x, y}(t), \mathbf{v}_{\mathbf{p}}, t\right) \\
0 & =G_{x, y}\left(\mathbf{z}_{x, y}(t), \mathbf{v}_{\mathbf{p}}, t\right)
\end{aligned}\right.
$$

where

$$
\mathbf{z}_{x, y}(t)=\left[\begin{array}{c}
z_{x, y, d}(t) \\
z_{x, y, a}(t)
\end{array}\right] .
$$

In the numerical simulations of the differential-algebraic systems in the form (6), the index of the system is of a great importance. In general, the algebraic part of the systems (6) can be differentiated according to the independent variable $t$

$$
\begin{aligned}
\dot{z}_{d}(t) & =F\left(z_{d}, z_{a}, \mathbf{v}_{\mathbf{p}}, t\right) \\
0 & =G\left(z_{d}, z_{a}, \mathbf{v}_{\mathbf{p}}, t\right)
\end{aligned}
$$

and as result the following form can be obtained

$$
\begin{aligned}
\dot{z}_{d}(t) & =F\left(z_{d}, z_{a}, \mathbf{v}_{\mathbf{p}}, t\right) \\
\frac{\partial G}{\partial z_{d}} \dot{z}_{d}+\frac{\partial G}{\partial z_{a}} \dot{z}_{a} & =-G\left(z_{d}, z_{a}, \mathbf{v}_{\mathbf{p}}, t\right)
\end{aligned}
$$

Definition 2.1: A differential-algebraic system (6) has an index one, if it can be rewritten as an ODE after exactly one differentiation.

Definition 2.2: A system of ordinary differential equations (ODEs) has an index zero.
To solve an index-one DAE system a vector of consistent initial conditions need to be known.

Definition 2.3: For the system (6) with the vector of the consistent initial conditions

$$
\mathbf{z}_{x, y}\left(t_{0}\right)=\left[\begin{array}{c}
z_{x, y, d}\left(t_{0}\right) \\
z_{x, y, a}\left(t_{0}\right)
\end{array}\right]
$$

the equation

$$
0=G_{x, y}\left(z_{x, y, d}\left(t_{0}\right), z_{x, y, a}\left(t_{0}\right), \mathbf{v}_{\mathbf{p}}, t_{0}\right)
$$

is fullfilled.

Application of these equations to model thermal phenomena has the following advantages

- the algebraic equations typically describe conservation laws or explicit equality constraints,

- it may be difficult or impossible to reformulate the model as an ODE when nonlinearities are present,

- the implicit models do not require the modeling simplifications often necessary to get an ODE;

- it is easier to vary design parameters in an implicit model,

- the variables keep their original physical interpretation [3].

In some practical applications an additional type of constraints need to be considered - the variability constraints. Especially, in such technological processes like a metal annealing or control of airplane, the observed changes cannot happen to fast, as well as to slow [1]. The variability constraints are imposed on the left-hand side of the ordinary differential equations and take the following form

$$
\dot{z}_{d}(t)<c_{v a r}(t)
$$

where $z_{d}(t)$ denotes the differential state variables and $c_{v a r}(t)$ represents the constraint function. The function $c_{v a r}(t)$ can take any appropriate form. In the literature the variability constraints have been formally introduced in [6]. Till now the variability constraints have been treated unformally using the right-hand side of the ODEs model

$$
F\left(z_{d}, z a, \mathbf{v}_{\mathbf{p}}, t\right)<c_{v a r}(t) .
$$

The representation (16) introduces all difficulties connected with the considered nonlinear differential model into a nonlinear optimization task.

The surface is consisted on the assumed number of cells $c_{x, y}(t)$. The temperature of each cell can be modeled by the system of continuous differential-algebraic equations (eq. 6). Moreover, to simulate the cell behavior, the consisted initial conditions need to be known, eqs. (13)-(14). The constraints, which represents the initial conditions, have a pointwise nature. Finally, the model optimization problem can be extended by the explicitly imposed variability constraints eq. (15). The presented methodology results in the nonlinear optimization task with the piecewise-continuous constraints. Therefore, the 
presented method is aimed at minimization of the objective function (2) subject to the presented constraints.

\section{THE OPTIMIZATION PROCEDURE}

The structure of the nonlinear optimization task, as well as an optimization procedure, is dependent on the discretization of the considered model-optimization problem. The new optimization-based procedure takes a form of a five step procedure.

1) The process duration time

$$
t \in\left[\begin{array}{ll}
t_{0} & t_{F}
\end{array}\right]
$$

is divided into given number $N$ subintervals. The length of each subinterval is equal $\Delta t$ and the duration of each interval is equal or larger than a measurements frequency

$$
\Delta t \geq \delta t
$$

where $\delta t$ is the measurements frequency. Therefore, the following relation can be observed

$$
t_{0}<t_{1}<t_{2}<\ldots<t_{N-1}<t_{F}
$$

where

$$
t_{n}=t_{0}+n \cdot \Delta t
$$

with $n=0,1, \cdots, N$.

2) The model discretization can be executed according to the obtained subintervals. The cell $c_{x, y}(t)$ can be represented by a series of submodels $c_{x, y}^{n}\left(t^{n}\right)$, $n=0,1, \cdots, N$, where

$$
\begin{aligned}
\dot{z}_{x, y, d}^{n}\left(t^{n}\right) & =F_{x, y}^{n}\left(z_{x, y, d}^{n}\left(t^{n}\right), z_{x, y, a}^{n}\left(t^{n}\right), \mathbf{v}_{\mathbf{p}}, t^{n}\right) \\
0 & =G_{x, y}^{n}\left(z_{x, y, d}^{n}\left(t^{n}\right), z_{x, y, a}^{n}\left(t^{n}\right), \mathbf{v}_{\mathbf{p}}, t^{n}\right)
\end{aligned}
$$

and

$$
t^{n}=\left[\begin{array}{ll}
t_{0}^{n} & t_{F}^{n}
\end{array}\right]
$$

3) The obtained measurements $m_{x, y}(t)$ represent the state of each cell $c_{x, y}$ at the time $t$. Moreover, in practical applications the process can be influenced by additional parameters. This remark indicates, that an external vector-valued control function $u(t)$ can be also under considerations.

4) Model parameters optimization. At this stage the unknown model parameters need to be identified. The identification can be treated as a model parameters optimization according to the obtained measurements

$$
\min _{\mathbf{v}_{\mathbf{p}}} \sum_{i=1}^{n_{x}} \sum_{j=1}^{n_{y}} \sum_{n=1}^{N}\left(c_{i, j}\left(t^{n}\right)-m_{j, j}\left(t^{n}\right)\right)^{2} .
$$

subject to the piecewise-continuous differentialalgebraic constraints. In some cases, the chosen cells can be can be characterized by the different properties.
Therefore, the extended vector of the model parameters need to be defined

$$
\mathbf{V}=\left[\begin{array}{c}
\mathbf{v}_{\mathbf{p}^{1,1}} \\
\vdots \\
\mathbf{v}_{\mathbf{p}^{1, n_{y}}} \\
\mathbf{v}_{\mathbf{p}^{2,1}} \\
\vdots \\
\mathbf{v}_{\mathbf{p}^{2, n_{y}}} \\
\vdots \\
\mathbf{v}_{\mathbf{p}^{n_{x}, 1}} \\
\vdots \\
\mathbf{v}_{\mathbf{p}^{n_{x}, n_{y}}}
\end{array}\right]
$$

where in $\mathbf{v}_{\mathbf{p}^{a, b}}: a=1, \cdots, n_{x}$ and $b=1, \cdots, n_{y}$.

5) The aim of the optimization algorithm is to find such values of the surface temperature distribution model $\mathbf{V}$, which could result in a desired state of the observed process. The presented discretization procedure enables us to obtain the discrete form of the differential-algebraic model constraints and pointwise constraints representing the initial conditions. Finally, all the obtained constraints with the unknown consistent initial conditions can be represented in a short form of the nonlinear optimization problem

$$
\min _{\mathbf{V}} \sum_{i=1}^{n_{x}} \sum_{j=1}^{n_{y}} \sum_{n=1}^{N}\left(c_{i, j}\left(t^{n}\right)-m_{j, j}\left(t^{n}\right)\right)^{2}=\min _{\mathbf{V}} f(\mathbf{V})
$$

subject to

$$
\begin{gathered}
c_{E}(\mathbf{V})=0 \\
c_{I}(\mathbf{V})-s=0 \\
s \geq 0 .
\end{gathered}
$$

The KKT conditions for the nonlinear optimization task in the form (25)-(28) can be presented as

$$
\begin{aligned}
\nabla f(\mathbf{V})-A_{E}^{T}(\mathbf{V}) \lambda_{E}-A_{I} \lambda_{I} & =0 \\
S \lambda_{I}-\mu e & =0 \\
c_{E}(\mathbf{V}) & =0 \\
c_{I}(\mathbf{V})-s & =0 \\
s & \geq 0 \\
\lambda_{I} & \geq 0
\end{aligned}
$$

where $A_{E}(\mathbf{V})$ and $A_{I}(\mathbf{V})$ are the Jacobian matrices of the functions $c_{E}$ and $c_{I}$, respectively. Moreover, $\lambda_{E}$ and $\lambda_{I}$ are their Lagrange multipliers. $S$ is the diagonal matrix with diagonal entries given by the vector $s$, and let $e=(1,1, \cdots, 1)^{T}$ [16]. To solve the KKT system, 
the interior-point optimization algorithm implemented in fmincon procedure [13] was used.

In the next section the results of the performed experiment, as well as the numerical computations, were presented.

\section{THE EXPERIMENTAL RESULTS}

The experiment has been performed with a metal key, which was a part of an electrical circuit. The voltage was equal to $22 \mathrm{~V}$. The key is an asymmetrical object and built from different layers. Each layer is characterized by another properties. The measurement interval was equal to $30 \mathrm{sec}$. The obtained results were presented on the Fig. 2-6.

There are two cells, which have been modeled by the proposed methodology. The applied model for each of the cells Sp1 and Sp2, had the general structure

$$
\dot{z}(t)=\mathbf{v}_{\mathbf{p}, \mathbf{1}} z(t)+\mathbf{v}_{\mathbf{p}, \mathbf{2}} u .
$$

In order to preserve the too fast changes of the object temperature, it was assumed that

$$
-10 \leq \dot{z}(t) \leq 10 \quad\left[{ }^{\circ} \mathrm{C} / \mathrm{sec}\right]
$$

Finally, the optimized model parameters of the assumed model function are as follows

$$
\dot{T}_{S p 1}(t)=-42.0085 \cdot T_{S p 1}(t)+48.1760 \cdot 22
$$

and

$$
\dot{T}_{S p 2}(t)=-38.9154 \cdot T_{S p 2}(t)+73.5950 \cdot 22 .
$$

The obtained results indicate two important questions, which are open for the future research:

1) The number of possible cells is really huge. The important question is, how to use efficient parallelization methods to applied the presented methodology in a reallife optimization algorithms? [7]

2) In order to minimize the number of the obtained models, new cells aggregation procedures should be designed [8].

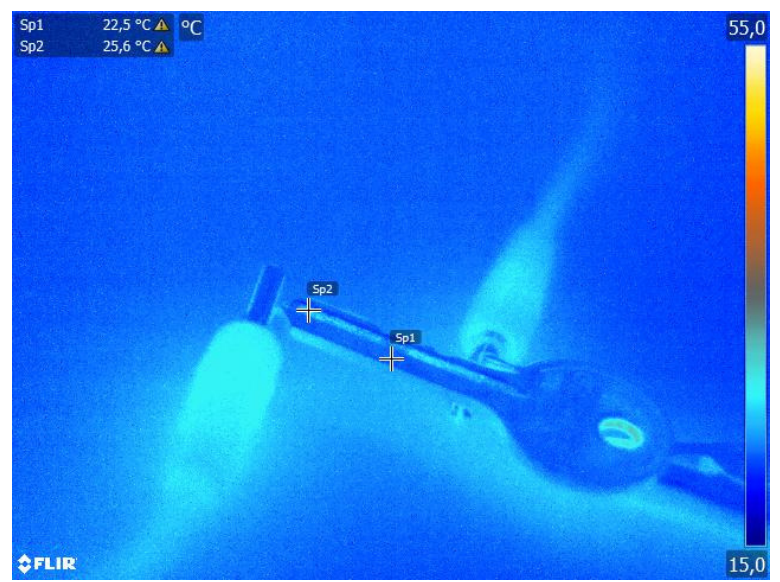

Fig. 2. The state of the object at the beginning of the process

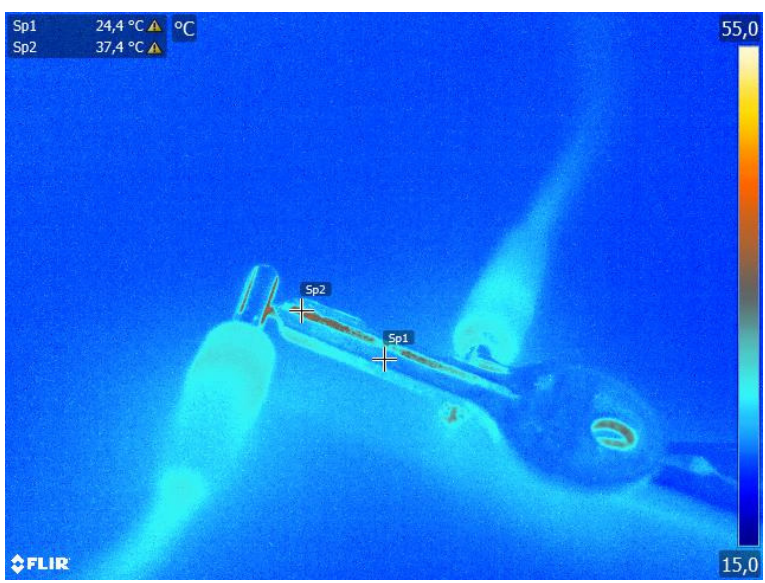

Fig. 3. The state of the object after $30 \mathrm{sec}$.

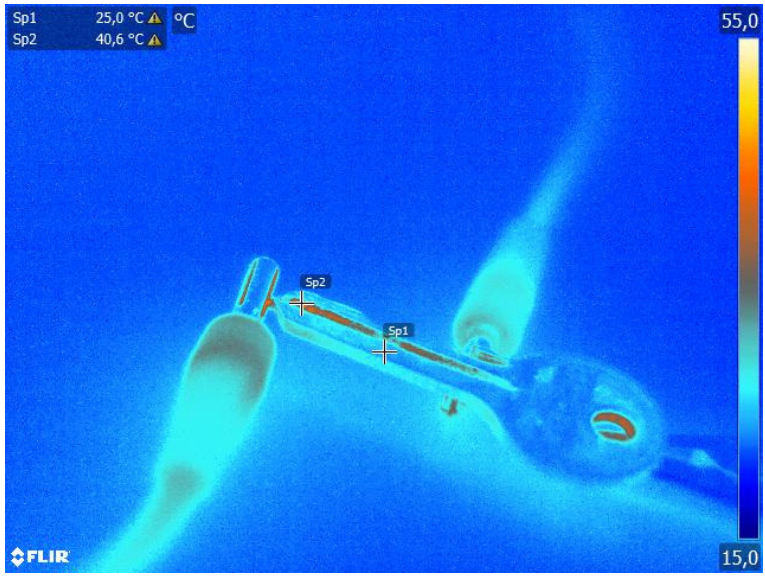

Fig. 4. The state of the object after $60 \mathrm{sec}$.

\section{CONCLUSION}

In the article a general optimization-based method for thermal processes modeling has been presented. The discussed methodology base on the spatio-temporal discretization of the measured process. To solve the obtained nonlinear optimization problem, the interior-point optimization algorithm was applied. The obtained results suggest two direction of the future work

- How to make the needed simulations in parallel in order to minimize a computation time?

- How to aggregate the spaces characterized by the similar properties in order to minimize the number of considered cell models?

\section{ACKNOWLEDGMENT}

This work has been supported by the National Science Center under grant: DEC-2012/07/B/ST7/01216

\section{REFERENCES}

[1] J.T. Betts. 2010. Practical Methods for Optimal Control and Estimation Using Nonlinear Programming, Second Edition. SIAM, Philadelphia, http://dx.doi.org/10.1137/1.9780898718577 


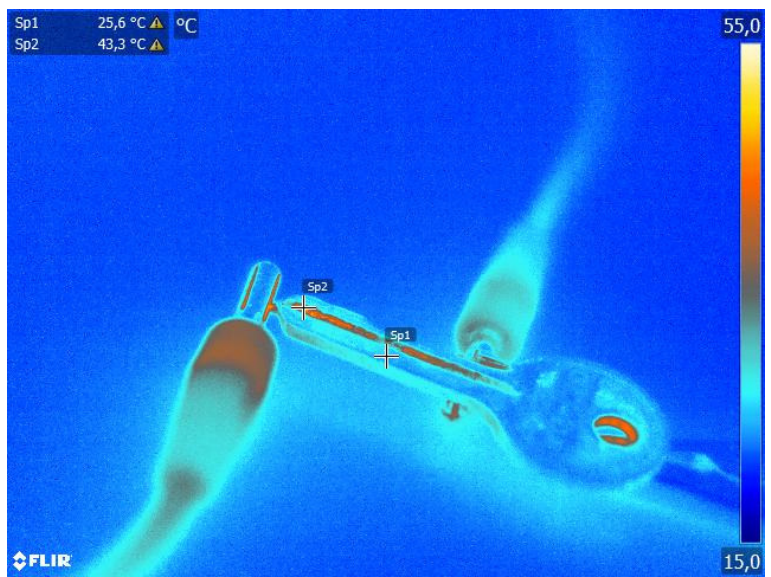

Fig. 5. The state of the object after $90 \mathrm{sec}$.

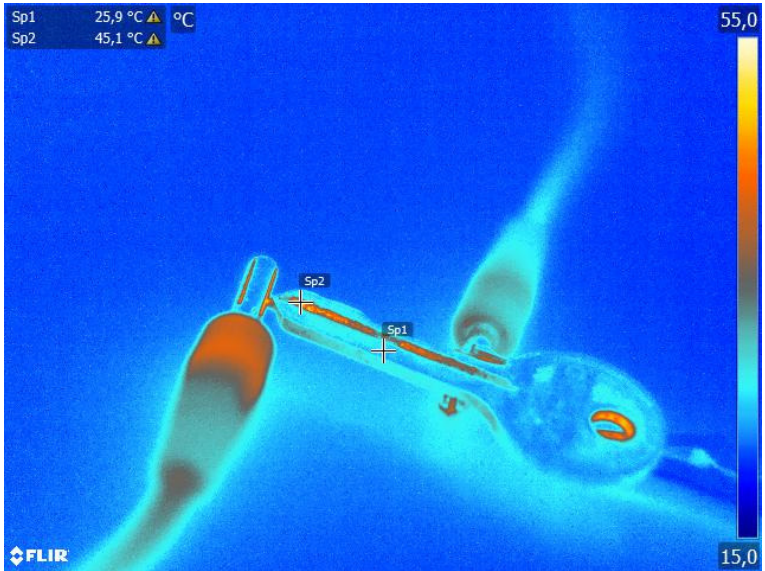

Fig. 6. The state of the object after $120 \mathrm{sec}$.

[2] D. Bhaduri, P. Penchev, A. Batal, S. Dimov, S.L. Soo, S. Sten, U. Harrysson, Z. Zhang, H. Dong. 2017. Laser polishing of 3D printed mesoscale components. Applied Surface Science. 405:29-46. http://dx.doi.org/10.1016/j.apsusc.2017.01.211

[3] L.T. Biegler, S. Campbell, V. Mehrmann. 2012. DAEs, Control, and Optimization. Control and Optimization with Differential-Algebraic Constraints. SIAM, Philadelphia, http://dx.doi.org/10.1137/9781611972252.ch1

[4] Y. Cao, A. Seth, C.D. Laird. 2016. An augmented Lagrangian interior-point approach for large-scale NLP problems on graphics processing units. Computers and Chemical Engineering. 85:76-83. http://doi.org/10.1016/j.compchemeng.2015.10.010

[5] F.E. Curtis, N.I.M. Gould, D.P. Robinson, P.L. Toint. 2017. An interior- point trust-funnel algorithm for nonlinear optimization. Mathematical Programming. 161:73-134. http://dx.doi.org/10.1007/s10107-016-1003-9

[6] P. Drag. 2016. Algorytmy sterowania wielostadialnymi procesami deskryptorowymi. Akademicka Oficyna Wydawnicza EXIT, Warszawa (in polish).

[7] P. Dragg, K. Styczeń. 2012. Parallel Simultaneous Approach for optimal control of DAE systems. 2012 Federated Conference on Computer Science and Information Systems, FedCSIS 2012. 517-523.

[8] P. Drạg, K. Styczeń. 2016. The constraints aggregation technique for control of ethanol production. Studies in Computational Intelligence. 655:179-192. http://dx.doi.org/10.1007/978-3-319-40132-4_11

[9] M. Fazlyab, S. Paternain, V.M. Preciado, A. Ribeiro. 2016. Interior Point Method for Dynamic Constrained Optimization in Continuous Time. 2016 American Control Conference (ACC), Boston Marriott Copley Place, July 6-8, 2016. Boston, MA, USA. 5612 -5618. http://doi.org/10.1109/ACC.2016.7526550

[10] E. Klintberg, S. Gros. 2016. An inexact interior point method for optimization of differential algebraic systems. Computers and Chemical Engineering. 92:163-171. http://doi.org/10.1016/j.compchemeng.2016.04.013

[11] Z. Li, T.C.E. Janssen, K.A. Buist, N.G. Deen, M. van Sint Annaland, J.A.M. Kuipers. 2017. Experimental and simulation study of heat transfer in fluidized beds with heat production. Chemical Engineering Journal. 317:242-257. http://dx.doi.org/10.1016/j.cej.2017.02.055

[12] F. Mariani, F. Risi, C.N. Grimaldi. 2017. Separation efficiency and heat exchange optimization in a cyclone. Separation and Purification Technology. 179:393-402. http://dx.doi.org/10.1016/j.seppur.2017.02.024

[13] MathWorks. 2017. Global Optimization Toolbox. User's Guide R2017a.

[14] J. Mei, X. Xia. 2017. Energy-efficient predictive control of indoor thermal comfort and air quality in a direct expansion air conditioning system. Applied Energy. 195:439-452. http://dx.doi.org/10.1016/j.apenergy.2017.03.076

[15] M. Mewa-Ngongang, H.W. du Plessis, U.F. Hutchinson, L. Mekuto, S.K.O. Ntwampe. 2017. Kinetic modelling and optimisation of antimicrobial compound production by Candida pyralidae KU736785 for contro of Candida guilliermondii. Food Science and Technology International 23:358-370. http://dx.doi.org/10.1177/1082013217694288

[16] J. Nocedal, S. Wright. 2006. Numerical Optimization. Springer, http://dx.doi.org/10.1007/978-0-387-40065-5

[17] E. Rafajłowicz, K. Styczeń, W. Rafajłowicz. 2012. A modified filte SQP method as a tool for optimal control of nonlinear systems with spatio-temporal dynamics. International Journal of Applied Mathematics and Computer Science. 22:313-326. http://dx.doi.org/10.2478/v10006012-0023-8

[18] A. Shinde, S. Arpit, P. KM, P.V.C. Rao, S.K. Saha. 2017. Heat Transfer Characterization and Optimization of Latent Heat Thermal Storage System Using Fins for Medium Temperature Solar Applications. Journal of Solar Energy Engineering. 139:031003. http://dx.doi.org/10.1115/1.4035517

[19] W. Uribe-Soto, J.-F. Portha, J.-M . Commenge, L. Falk. 2017. A review of thermochemical processes and technologies to use steelworks off-gases. Renewable and Sustainable Energy Reviews. 74:809-823. http://dx.doi.org/10.1016/j.rser.2017.03.008

[20] L. Wang, X. Liu, Z. Zhang. 2017. An efficient interior-point algorithm with new nonmonotone line search filter method for nonlinear constrained programming. Engineering Optimization. 49:290-310 http://dx.doi.org/10.1080/0305215X.2016.1176828

[21] V.I. Zorkaltsev. 2016. Search for feasible solutions by interio point algorithms. Numerical Analysis and Applications. 9:191-206. http://doi.org/10.1134/S1995423916030022 